\title{
Measuring SEDs for individual galaxy components
}

\author{
Steven P. Bamford ${ }^{1}$, Boris Häußler ${ }^{1}$, \\ Alex Rojas ${ }^{2}$, Marina Vika ${ }^{2}$ and Jim Cresswell ${ }^{2}$ \\ ${ }^{1}$ School of Physics and Astronomy, University of Nottingham, \\ University Park, Nottingham, NG7 2RD, UK \\ email: steven.bamford@nottingham.ac.uk \\ ${ }^{2}$ Carnegie Mellon University in Qatar, PO Box 24866, Doha, Qatar
}

\begin{abstract}
Our project, 'MegaMorph', is developing a next-generation tool for decomposing galaxies, in terms of both their structures and stellar populations. By combining data from UV to NIR wavelengths, accounting for morphological peculiarities using non-parametric components, and utilising efficient likelihood sampling methods, we are working to significantly improve the robustness and accuracy of galaxy decomposition. Applying these new techniques to modern large surveys will provide us with a deeper understanding of galaxies.
\end{abstract}

Keywords. galaxies: fundamental parameters, galaxies: structure, galaxies: photometry, methods: data analysis, techniques: image processing

\section{Galaxy components}

Galaxies comprise a complex mix of stars, gas, dust and dark matter, with their proportions, distributions and kinematics resulting from a wide variety of physical processes. Even considering just the stars, galaxies typically possess distinct stellar populations, which formed at different times, under varied conditions. To gain more than a superficial understanding of the galaxy population, and the processes that shape it, we need to disentangle these physical components within individual galaxies.

This conference primarily focused on separating and characterising galaxy components through their spectral energy distributions. These SEDs are usually, with a few notable exceptions, integrated over the spatial extent of each galaxy. For separating components which dominate at different wavelength ranges, e.g., stars and dust, this approach works well. However, when dividing components with only subtle differences in their SEDs, e.g., multiple stellar populations, any additional discriminatory information is valuable.

The various stellar populations within a galaxy often have contrasting spatial distributions. In our simplest models, a galaxy grows through two principal mechanisms: the gradual accretion of gas, which cools and forms a thin disk of stars; and the merging of existing stellar systems, which produces a spheroid. These two structural components thus form through disparate mechanisms, contain different stellar populations, and represent disjoint periods in a galaxy's history (e.g., Cole et al. 2000; Cook et al. 2009; Benson 2010). Using information regarding both the spatial distributions and SEDs of these components will clearly enable a more robust separation of their properties, when compared with trying to use just one of these pieces of information alone. The dichotomy between disk and spheroid stellar components (e.g., Allen et al. 2006; Benson \& Devereux 2010) is at the heart of our understanding of galaxy development: measuring their individual properties will provide a deeper understanding of the galaxy population and more effective comparison with theoretical models. 


\section{Present structural decomposition solutions}

A number of solutions exist for decomposing galaxy structures (e.g., GALFIT, Peng et al. 2002, 2010; GIM2D, Simard et al. 2002, etc.), which generally fit smooth, parametric models to a single image of each galaxy. These have been applied to large samples (e.g., Allen et al. 2006; Simard et al. 2011), confirming the value of the approach. However, there remain significant problems regarding the robustness and physical interpretation of the fitted model parameters, e.g., Allen et al. find that $\sim 15$ per cent of their attempted bulge+disk fits result in unphysical parameters. These difficulties are due to degeneracies between the parameters of the two galaxy components in a single wavelength-band model, exacerbated by low signal-to-noise imaging, and the presence of features in the data (e.g., star-formation regions, spiral arms, bars, etc.) which are not included in the model.

A further issue is the measurement of SEDs for the separate components. Surveys now typically produce imaging with comparable quality across many photometric bands. For example, the SDSS (Abazajian et al. 2009) and UKIDSS LAS (Lawrence et al. 2007) surveys together provide imaging spanning nine optical and near-infrared bands. One may treat each band independently, but the recovered components will differ in size and shape and thus not correspond to the same population of stars at each wavelength, compromising the physical meaning of their colours. More usefully, one can perform a full fit in a single band, and then rescale the flux of the resulting model to fit each additional band. This produces meaningful colours for the physically distinct components, but their quality depends upon the reliability of the initial single-band decomposition. As mentioned above, a significant fraction of these will be physically unrealistic.

\section{Next-generation galaxy decomposition}

We have embarked on a project, named MegaMorph, which aims to improve our ability to separate galaxy components, particularly in large surveys. Our approach is to build upon an existing, tried-and-tested system which takes care of the numerous details that are essential for reliable galaxy fitting, but which do not require significant alteration. We therefore base our developments on GALAPAGOS (Häussler et al. 2007), which performs detection, deblending and preliminary object measurements (using SExtractor; Bertin \& Arnouts 1996), measures the sky level, extracts galaxy images, creates masks, fits each galaxy using GALFIT (Peng et al. 2002), and finally collates the results.

Below we describe the developments we are considering, in order to address the issues discussed in the previous section. This work is ongoing, but we have already made substantial progress implementing these methods, and are now focusing on testing and refining our techniques, while starting work on key science examples to demonstrate their practical benefits. We assess our modifications to GALAPAGOS and GALFIT using a variety of data, including: (1) nearby galaxies with SDSS imaging, artificially redshifted using FERENGI (Barden et al. 2008), (2) the Galaxy And Mass Assembly survey (Driver et al. 2009, 2010) which provides homogenised imaging over nine optical to near-infrared bands, redshifts, and a great deal of other data, together with visual morphologies from Galaxy Zoo (Lintott et al. 2010), and (3) simulations of the GAMA imaging created by extending the method of Häussler et al. (2007).

\subsection{Multiband fitting}

We propose that the reliability of the decomposition process can be greatly increased, while ensuring physically meaningful component colours, by constraining a single wavelength-dependent model using all of the available multiband imaging simultaneously. Firstly, this increases the signal-to-noise of the data used to constrain the fit, for a 

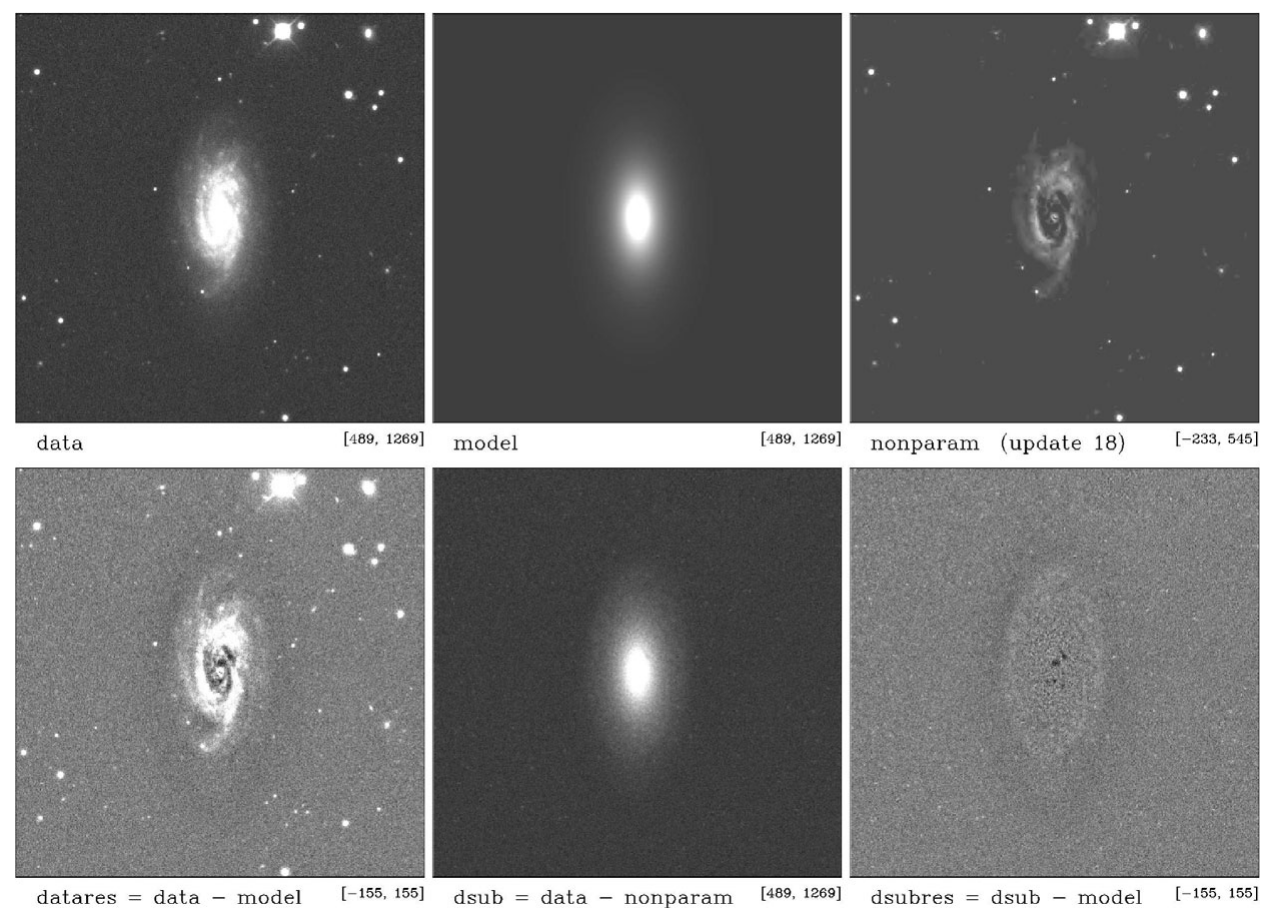

Figure 1. An example of fitting a spiral galaxy with a non-parametric component. The panels show (data) the original image; (model) the final parametric model, comprising a Sérsic bulge and a exponential disk; (nonparam) the non-parametric component; (datares) the residual of the original image minus the parametric model; (dsub) the original image minus the non-parametric component, note that the smooth profile, pixel noise and some low contrast features remain in this image; and (dsubres) the residual of the original image with the non-parametric component and parametric model subtracted, an unmodelled outer disk truncation is just visible. The numbers to the bottom right of each panel give the limits of the linear intensity scale.

comparatively small increase in the number of fitted parameters. Secondly, and more importantly, multi-wavelength imaging provides information that is not available to techniques which operate on only a single band at a time: the wavelength dependence of the flux of each component. We expect this to be critically important for overcoming the degeneracies inherent in multicomponent fitting, particularly in the common case of an exponential disk and Sérsic bulge. Finally, this approach enables the use of SED priors for each component, reducing the effective number of free parameters.

\subsection{Non-parametric components}

The observed surface brightness distribution of galaxies, particularly at blue wavelengths, is strongly modulated by star formation, dust and resonance structures such as spiral arms, bars and rings. However, in general, most of the stellar mass may be identified with a small number of relatively smooth, approximately azimuthally-symmetric components, and it is therefore these components which we primarily wish to characterise. The conventional approach is to fit idealised, smooth models to the images of real, messy, galaxies. However, it is generally unwise to fit data with a model which is unable to adequately represent the behaviour of that data, and then attempt to interpret the resulting parameters in a meaningful way (e.g., see Hogg et al. 2010).

To solve this dilemma, we are developing a technique which adds non-parametric components to the galaxy model, to account for features that are not represented by the 
usual parametric components. An exciting aspect of this work is the combination with the multiband approach, which enables one to fit for multiple non-parametric components with different colours. We have produced encouraging results so far, see Figure 1 , and we hope that this approach will substantially increase the reliability of the recovered parameters (and their uncertainties) for the spheroid and disk components of galaxies.

\subsection{Parameter uncertainties and model selection}

GALFIT uses the Levenberg-Marquardt algorithm to find the optimal model fit to the data. This is a downhill method, and therefore only samples a small number of points along the minimisation path. While this provides rapid convergence to the nearest local minimum, it does mean that the result may depend upon the starting parameters, that no knowledge of the overall likelihood landscape is acquired, and that the resulting parameter uncertainties are highly approximate.

In certain cases it is worth sacrificing speed for a better understanding of parameter degeneracies, accurate uncertainties, and an estimate of the Bayesian evidence. The latter helps select the most suitable model from a range of possibilities, e.g. single Sérsic, bulge+disk, etc. Obtaining all this extra information requires the parameter space to be more generally sampled, which, to be completed in an reasonable time, requires the use of efficient algorithms. One excellent example, well used in cosmology, is provided by the MultiNest (Feroz et al. 2009) library. We have incorporated this sampler as an option in GALFIT, thereby providing the additional details described above. The cost is a running time 100 - 1000 longer than usual, but this computational increase is surmountable, for reasonably large samples, through the use of supercomputers.

\section{Acknowledgements}

This work was made possible by a NPRP grant from the Qatar National Research Fund (a member of the Qatar Foundation). The statements made herein are solely the responsibility of the authors. SPB holds an STFC Advanced Fellowship.

\section{References}

Abazajian, K. N., et al. 2009, ApJS, 182, 543

Allen, P. D., et al. 2006, MNRAS, 371, 2

Barden, M., Jahnke, K., \& Häußler, B. 2008, ApJS, 175, 105

Benson, A. J. 2010, Phys. Rep., 495, 33

Benson, A. J. \& Devereux, N. 2010, MNRAS, 402, 2321

Bertin, E. \& Arnouts, S. 1996, A\&AS, 117, 393

Cole, S., et al. 2000, MNRAS, 319, 168

Cook, M., Lapi, A., \& Granato, G. L. 2009, MNRAS, 397, 534

Driver, S. P., et al. 2009, A\&G, 50, 5.12

Driver, S. P., et al. 2011, MNRAS, 413, 971

Feroz, F., Hobson, M. P., \& Bridges, M. 2009, MNRAS, 398, 1601

Häussler, B., et al. 2007, ApJS, 172, 615

Hogg, D. W., Bovy, J., \& Lang, D. 2010, ArXiv e-prints, arXiv:1008.4686

Lawrence, A., et al. 2007, MNRAS, 379, 1599

Lintott, C., et al. 2011, MNRAS, 410, 166

Peng, C. Y., et al. 2002, AJ, 124, 266

Peng, C. Y., et al. 2010, AJ, 139, 2097

Simard, L., et al. 2002, ApJS, 142, 1

Simard L., et al. 2011, ApJS, 196, 11 


\section{Discussion}

BUREAU: If I may make a comment, decomposing galaxies into independent components is fine as a practical/numerical tool, but one should be careful about assigning a physical meaning and interpretation. Galaxies are not static objects with stars as building blocks but dynamic objects with orbits as building blocks. So separating a galaxy into e.g. a disk and a bulge creates two entities that could not exist separately. Especially, bulges are rarely "mini-ellipticals" but mostly disk phenomena: thickened bars ("peanut-shaped" bulges) and disk-like ("pseudo") bulges. So associating steep light profiles (from bulgedisk photometric decompositions) with spheroids is fraught with danger and can lead to flawed interpretations. Use with caution! 